International Archives of the Photogrammetry, Remote Sensing and Spatial Information Sciences, Volume XL-1/W1, ISPRS Hannover Workshop 2013, 21 - 24 May 2013, Hannover, Germany

\title{
EUFODOS
}

\section{European Forest Downstream Services - Improved Information on Forest Structure and Damage}

\author{
M. Hirschmugl, H. Gallaun, R. Wack, K. Granica, M. Schardt \\ JOANNEUM RESEARCH, DIGITAL, Dep. for Remote Sensing and Geoinformation, 8010 Graz, Austria \\ manuela.hirschmugl@joanneum.at
}

Commission VI, WG VI/4

KEY WORDS: LiDAR, airborne laser-scanning, EUFODOS, forest parameters, protective forest, tree height, tree tops.

\begin{abstract}
:
Forests play a key role in the European economy and environment. This role incorporates ecological functions which can be affected by the occurrence of insect infestations, forest fire, heavy snowfall or windfall events. Local or Regional Authorities (LRAs) thus require detailed information on the degradation status of their forests to be able to take appropriate measures for their forest management plans. In the EUFODOS project, state-of-the-art satellite and laser scanning technologies are used to provide forest authorities with cost-effective and comprehensive information on forest structure and damage. One of the six test sites is located in the Austrian province of Styria where regional forest authorities have expressed a strong need for detailed forest parameters in protective forest. As airborne laser-scanning data is available, it will be utilized to derive detailed forest parameters such as the upper forest border line, tree height, growth classes, forest density, vertical structure or volume. At the current project status, the results of (i) the forest border line, (ii) the segmentation of forest stands and (iii) the tree top detection are available and presented including accuracy assessment and interim results are shown for timber volume estimations. The final results show that the forest border can be mapped operationally with an overall accuracy of almost 99\% from LiDAR data. For the segmentation of forest stands, a comparison of the automatically derived result with visual-manual delineation showed in general a more detailed segmentation result, but for all visual-manual segments a congruence of $87 \%$ within a $4 \mathrm{~m}$ buffer. Tree top detections were compared to stem numbers estimated based on angle-count samplings in a field campaign, which led to a correlation coefficient (R) of 0.79 .
\end{abstract}

\section{INTRODUCTION}

The purpose of GMES is to deliver information that meets the users' needs, thus the Forest Services being developed within EUFODOS are strongly focused on this aspect. EUFODOS involves an extensive user community well connected to other related GMES User Groups. The EUFODOS project aims to develop required Forest Downstream Services in an economically viable manner. The services encompass the assessment of forest damage and the measurement of functional parameters for protective forests, because these monitoring services are urgently required by regional forest authorities. They are being developed by a consortium of research organizations, commercial service providers and users from Austria, Bulgaria, Finland, Germany, Italy and Poland. In the Austrian province of Styria an ALS (airborne laser-scanning, LiDAR) survey campaign has been initialized during the years 2008-2012. This data type opens new opportunities for the Styrian Forestry Board to obtain forest data over large regions often covered with protective forests.

\subsection{Objectives}

In the elaboration of the user requirements of EUFODOS a parameter catalogue was created consisting of the following parameters: species type, density, growth classes, forest border, tree height, vertical stand structure, crown volume, understorey vegetation and natural regeneration. The EUFODOS objective therefore is to analyse the feasibility how these forest variables can be derived from ALS data and assess the related accuracy. Consecutively an operational service is set up in order to produce these parameters over large regions in an economically viable manner. In the current paper the focus is put on the delineation of the forest and shrub-land border, detection of tree tops as well as the segmentation process, and on the stand-wise estimation of timber volume.

\subsection{State of the Art}

There is a wide range of papers on forest parameters from LiDAR data, both on forest stand and individual tree level, available. A review on can be found in Hyyppä et al. (2009). One of the first investigations on LiDAR has been Nelson et al. (1988). Many authors proved that information related to height or structure of forests as well as biomass can be extracted with high quality from LiDAR data (Koch 2010). Lefsky et al. (2001) explained $84 \%$ of the aboveground biomass variance by regression from the LiDAR measured canopy structure. For biomass estimation from LiDAR data, the indirect approach is often chosen, that means tree heights are first calculated and then wood volume is modelled on this basis (Straub et al., 2009) and finally expansion factors are applied to estimate biomass. Popescu (2007) managed to explain $93 \%$ of the biomass using individual tree metrics. Wack and Stelzl (2005) reached an $\mathrm{R}^{2}$ of 0.94 for timber volume and dominant height and 0.9 for stem numbers. Järnstedt et al. (2012) have shown the great potential of LiDAR data for the derivation of forest parameters, such as number of trees, mean height, basal area or volume of growing stock, for a study area in Finland. Alberti et al. (2013) investigated the use of LiDAR data as support for the characterization of structure, volume, biomass and naturalistic value in mixed-coniferous forests of the Alpine region. Volume and biomass were then computed using regression models. Strong correlations $\left(\mathrm{R}^{2}=0.8\right)$ between LiDAR height and ground-measured volume were detected. 


\section{DATA \& TEST AREA}

The basic data is LiDAR data obtained by airborne survey in 2008. The data was obtained by a Riegl LMS-Q560 sensor with $200 \mathrm{kHz}$ and the original point cloud has a density of 4 points $/ \mathrm{m}^{2}$ below $2000 \mathrm{~m}$ a.s.l. and 2 points $/ \mathrm{m}^{2}$ above this elevation limit. For this study, an area of $10 \times 10 \mathrm{~km}$ in Hohentauern was selected stretching from 1100 to $2200 \mathrm{~m}$ a.s.l. The test region is mainly stocked with coniferous trees, spruce and larch, which are in the subalpine tree line mixed with dwarf mountain pine and green alder. Aside from the LiDAR data, also CIR orthoimages from 2011 were available for visual interpretation and comparisons. A field measurement campaign was performed in summer 2012 to obtain ground truth data. 51 forest stands were visited and for each, between three and four angle-count samplings were measured depending on the homogeneity of the spatial and vertical structure. The diameter and breast height was measured by calliper and the tree species was recorded. In addition tree heights are measured for both the strongest and median tree of each tree type within one anglecount sample using a Vertex-III measurement unit. Based on this input data, the timber volume was calculated using standard calculation methods from forest research. It has to be mentioned, that there is a significant time difference between the LiDAR data acquired in 2008 and the field information obtained in 2012. Therefore three stands had to be eliminated from the analysis leading to 48 forest stands with field measured attributes.

\section{METHODS}

The workflow of the EUFODOS processing line encompass all necessary steps for the derivation of the forest parameters from the raw LiDAR point cloud to the resulting forest parameter map. The most important processing steps are described below.

\subsection{Delineation of the Forest Border}

Accurate delineation of the forest border in a mountainous environment is a critical task, because the forest border is a fundamental input for a broad range of applications. A main requirement for the delineation of the forest border is that it is based on clear nomenclature definitions. As nomenclature definitions vary at the national and international level as well as for the specific field of application, especially on the upper forest border, the developed method allows flexible specification of the main parameters "minimum forest area" "minimum non-forest area", "minimum distance between stands", "minimum tree height" and "minimum crown cover". Whereas standard segmentation approaches do not allow defining these parameters as segmentation constraints, the developed approach provides a delineation which straight forward follows these parameters. In the following, the main processing steps are described.

First, from the LIDAR data, a nDSM (normalized digital surface model) is derived as the difference between the digital surface model and the digital elevation model, and settlements are automatically excluded by applying morphological operations based on the detected buildings, and gap filling is performed. Secondly, crown cover is calculated over segments with a minimum size according to the specified "minimum forest area" parameter. The crown cover is thereby defined as the proportion of the forest floor covered by the vertical projection of the tree crowns over the respective segments. Within those areas which show a crown cover above the specified "minimum crown cover" parameter, aggregation according to the specified "minimum distance between stocked areas" parameter is applied. Then, generalization is performed according to the "minimum stocked area" and "minimum unstocked area” parameters by applying standard morphological operations. As result of these automatic processing steps, stocked areas are delineated according to the defined parameters based on LIDAR data only.

Often the forest area definition requires taking into account further nomenclature constraints, such as for example, incorporation of temporarily un-stocked areas into forest land or exclusion of tree covered areas according dominant land-use (e.g. parks, orchards, arable land with trees). To incorporate such additional constraints into the generalization process, additional generalization steps could be applied by integrating also additional information (e.g. classification of multi-temporal satellite imagery), or by visual refinement of the automatically derived generalization results.

\subsection{Tree Top Detection}

The TreeTop Detector, implemented in IMPACT, automatically detects tree tops based on a LiDAR nDSM as input. The method uses a multi-scale Laplacian of Gauss (LoG) method, which is a combination of Laplacian and Gauss filter (Gonzalez and Woods, 2002; Hirschmugl, 2008). The filtering is used to blur the image being determined by the value of the standard deviation $(\sigma)$. In order to detect trees of different sizes three different $\sigma$ values are applied to the LoG filtering and consecutively combined (see Figure 1). The summation image from the LoG filtering is then used to detect the intensity maxima by using a local maximum approach. As a result of this process the tree tops including their height values are derived. The most important advantage of this method compared to previous methods is its independence from tree models, i.e. no a-priori information about tree species is necessary.
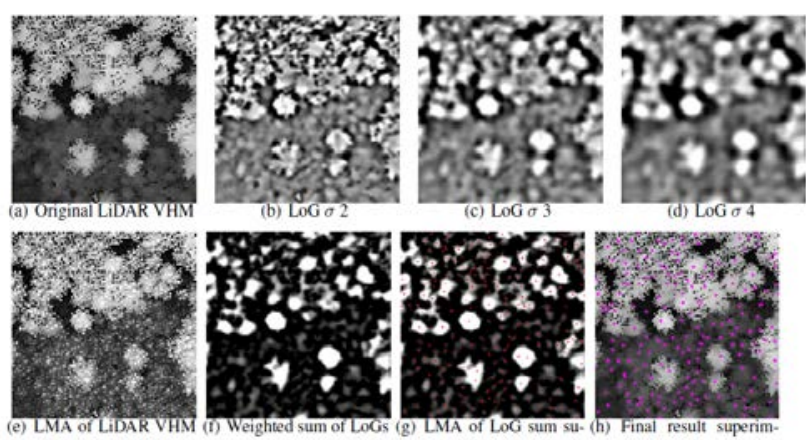

superimposed on VHM

perimposed on LoG sum posed on VHM

Figure 1. Processing steps and intermediate results for the LoG approach based on LiDAR nDSM data.

\subsection{Segmentation of Forest Stands}

Within the defined forest area (from Chapter 3.1), a segmentation procedure is applied to divide the forest into relatively homogeneous forest segments or forest stands. The segmentation builds mainly on the nDSM, but additional optional inputs are optical data and/or forest roads. The resulting forest segments should show relatively homogeneous tree height and vertical forest structure. In order to ensure similar tree height, the nDSM (normalized digital surface model) is used, for assessing the vertical stand structure, a 
minimum variance wedge filter is calculated as a textural parameter. These two data sets are then stacked to one image and used in a three-step region growing image segmentation procedure to derive polygons of homogeneous forest segments. The first step is a coarse segmentation, which derives the main skeleton of segments. This coarse segmentation uses the input files down-sampled to $5 \mathrm{~m}$ spatial resolution and smoothed by symmetric nearest neighbor (SNN) filter. The result shows the main borders, but the outline is not exact with regards to the tree crown outlines. In a second step, a fine-segmentation is performed using the full resolution data and resulting in a clear over-segmentation of the forest, partly with ring-like results, but representing a very exact delineation along the tree crowns. In the final third step, the two results are combined by snapping the coarse segment borders to the fine segmentation outlines. This is done using the ArcGIS Tool 'update', the effect is visualized in Figure 2. The orange lines are the coarse segment borders and the blue lines are the fine segment borders. After the combination, the resulting borders (red) are refined as long as the distance to the original line is below a user defined threshold, which was set to one meter in the current case. This outline refinement is necessary in order to avoid errors in the forest parameter calculation, especially for the parameter 'height of dominant layer'. The final forest segments (see Figure 3) are then used to calculate the respective forest parameters within each polygon.

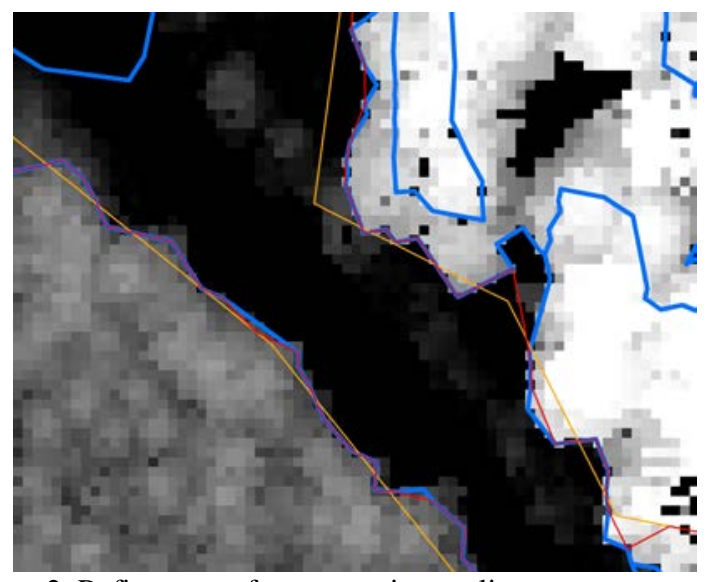

Figure 2. Refinement of segmentation outlines: orange $=$ coarse segmentation, blue $=$ fine segmentation, red $=$ combined result.

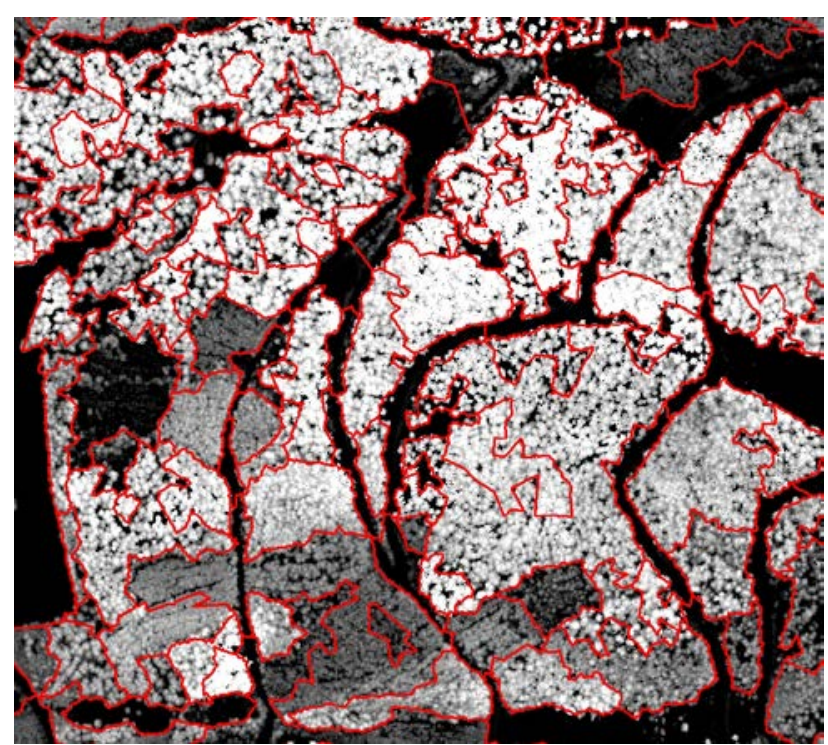

Figure 3. Segmentation result of forest stands based on nDSM.

\subsection{Derivation of Forest Parameters}

Various forest parameters are needed for the proper management of protective forests. In the following methods are described for several height parameters and timber volume.

Based on the tree top detections, three different forest stand height values are calculated: height of upper layer (= mean height of $20 \%$ highest trees per segment), mean height (= mean height of all detected trees) and height of second layer, if existing (= mean height of the $20 \%$ highest trees of the second layer, which is defined as trees smaller than $2 / 3$ of the height of upper layer). The three height parameters are visualized in Figure 4. These height parameters are on the one hand used for the calculation of timber volume and on the other hand are per se important attributes for the forest authorities to determine the condition of the forest. The existence of a second layer is for example stated to be important information for the forest authorities.

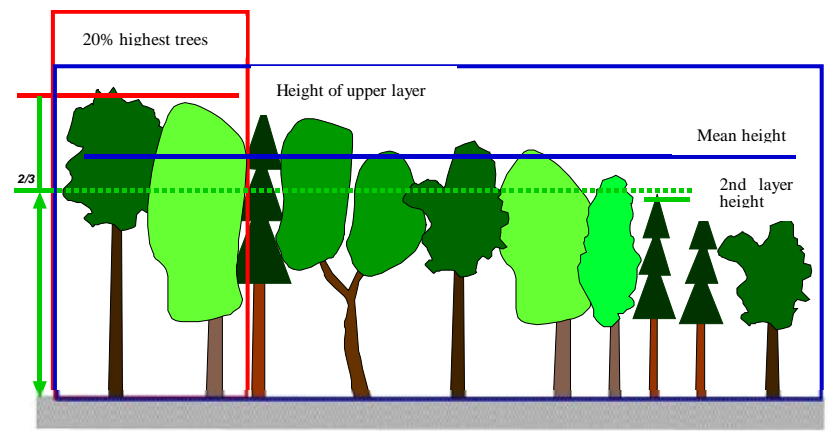

Figure 4. Derived height parameters.

For the estimation of timber volume, different variables have been tested. These are the different height values calculated based on the detected tree tops, canopy cover, and the so-called 'volume integral', which is defined as the sum of all vegetation heights in a forest stand.

\section{RESULTS \& ACCURACY}

In order to assess the quality of the results, comparisons with field data on the one hand (for tree top detection and timber volume) and with visual interpretation on the other hand (for forest border and segmentation) were performed. The individual results are given in the following sections.

\subsection{Results \& Accuracy of Forest Border}

The forest border has been derived according to the requirements of the user organisation "Styrian Forestry Board". Main focus of the user organisation within the current project was on delineation of the forest and shrub-land border at the subalpine tree line, where gradually changing crown cover occurs within a complex pattern of stocked areas, shrub-land and succession areas. They specified following parameters: Minimum crown cover of trees is $10 \%$ over areas with a minimum size of $1000 \mathrm{~m}^{2}$, minimum tree height above $1.3 \mathrm{~m}$, minimum distance between stocked areas is $10 \mathrm{~m}$, minimum stocked area of $1000 \mathrm{~m}^{2}$, and minimum un-stocked area of 1000 $\mathrm{m}^{2}$. Further, a separation of shrub-land (dwarf mountain pine, green alder stands and succession areas) was performed by applying the same generalisation procedure in addition with a "maximum tree height" of $5 \mathrm{~m}$. The automatically derived forest and shrub-land areas where then refined according to the user 
defined additional nomenclature specifications, e.g. exclusion of areas dominated by non-forest land-use and inclusion of temporarily un-stocked areas into forest land. The effort for the visual revision was five hours per $100 \mathrm{~km}^{2}$.

Quality control has been performed by random sampling with $\mathrm{n}=298$ sampling points. The forest and shrub-land areas were compared by visual interpretation of LIDAR and CIR aerial imagery according to above described nomenclature specifications (see Table 1). The result of the quality control shows a very high accuracy of the delineated forest and shrubland areas.

\begin{tabular}{|l|c|c|c|}
\hline & 0-noforest & 1-forest & UA [\%] \\
\hline 0-noforest & 90 & 0 & 100.00 \\
\hline 1-forest & 3 & 205 & 98.56 \\
\hline PA [\%] & 96.77 & 100.00 & \\
\hline Overall accuracy [\%] & 98.99 \\
\hline
\end{tabular}

Table 1. Accuracy assessment of the forest and shrub-land border.

\subsection{Results \& Accuracy of Tree Top Detection}

The tree detection result in an area of forest stands of different growth classes is shown in Figure 5 superimposed on the nDSM. For accuracy assessment, the result is compared to the stem numbers estimated from the field plots. It could not be compared to a full tree inventory, as the generation of such a full inventory of individual trees is very time consuming, especially in this alpine environment. The comparison with estimated stem numbers from the 48 stands surveyed in the field led to a coefficient of correlation (R) of 0.79 .

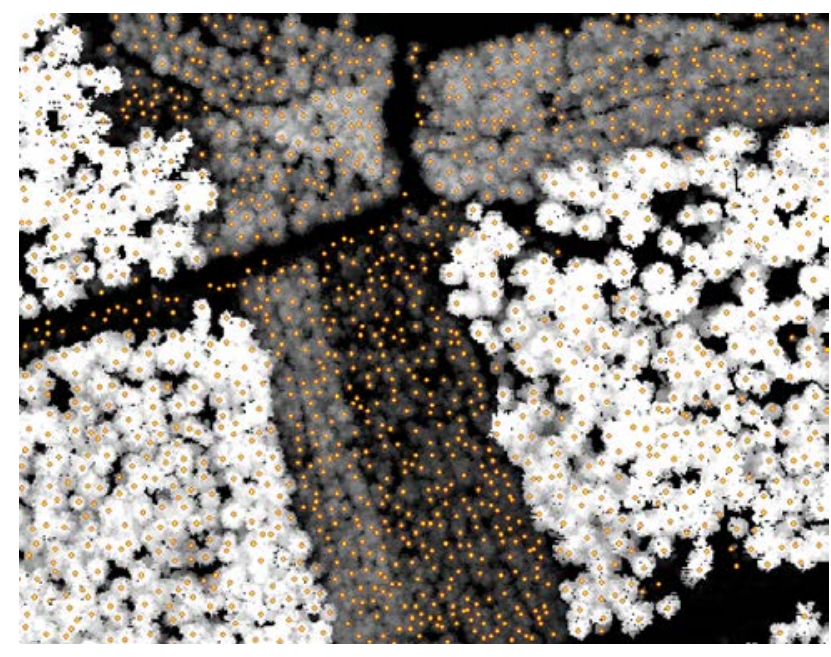

Figure 5. Tree top detection result.

\subsection{Results \& Accuracy of Segmentation}

The produced segments were compared to an independent visual-manual segmentation (reference segmentation) done by an experienced interpreter. Such a comparison is tricky, because two completely different approaches are used. Nonetheless, such a comparison is the only option to quantitatively assess the segmentation result. The reference segmentation covers an area of 322.5 ha of forest and led to $127.3 \mathrm{~km}$ segment borders in 533 segments. In comparison, the 990 automatically generated segments have a total length of $197.6 \mathrm{~km}$. The reasons for these differences are twofold. First, the automated segmentation produces smaller segments, especially in the areas of low canopy cover close to the upper forest border. Second, the automatic segmentation outline is due to the refinement much more detailed leading to an increased outline length. In order to assess the percentage of segment boundaries found by both the automatic and the reference segmentation, the reference segmentation was buffered. Using a buffer of $2 \mathrm{~m}$ led to $60 \%$ of the reference lines correctly found by the automatic segmentation. Increasing the buffer size to $4 \mathrm{~m}$ led to a detection rate of $87 \%$, all details can be found in Table 2. As already mentioned, the high commission error is mainly due to the fact, that the automatic segmentation leads to smaller segments as the ones typically produced by visual interpretation.

\begin{tabular}{|l|c|c|}
\hline & Buffer 2 m & Buffer 4 m \\
\hline Correct & $60 \%$ & $87 \%$ \\
\hline Commission error & $61 \%$ & $43 \%$ \\
\hline Omission error & $40 \%$ & $13 \%$ \\
\hline
\end{tabular}

Table 2. Comparison of automatic and reference segmentation.

\subsection{Preliminary Results of Timber Volume Estimation}

The correlation results with the timber volume estimated from the field measurements for 48 forest stands are given in Table 3 . The results are below the expected accuracy which has been reached on the one hand in the literature and on the other hand also in previous projects with the same methods. Therefore additional work is needed to analyse the results in detail. In particular, some of the field plots have to be re-visited to ensure highly accurate ground truth data. In this second visit, also signs of changes during the last years will be paid additional attention to, as there is a time difference of four years between LiDAR data and field data acquisition. A further reason for the relatively low correlation results might be growing conditions in this alpine forest habitat.

\begin{tabular}{|l|c|}
\hline Variable(s) & $\mathrm{R}$ \\
\hline Height of upper layer & 0.68 \\
\hline Mean height & 0.71 \\
\hline Canopy space integral & 0.77 \\
\hline Canopy space integral \& height of upper layer & 0.78 \\
\hline Canopy space integral \& mean height & 0.78 \\
\hline $\begin{array}{l}\text { Canopy space integral \& height of upper } \\
\text { layer*standard deviation of height }\end{array}$ & 0.80 \\
\hline
\end{tabular}

Table 3. Results of correlation with timber volume.

\section{DISCUSSION \& CONCLUSION}

The forest border derived from LiDAR data is highly accurate with almost $99 \%$ overall accuracy and therefore superior to previous forest masks generated from satellite and orthophoto data (Gallaun et al, 2007) with an overall accuracy of $96 \%$. As both are automatic approaches with one final visual correction step, the LiDAR data is to be preferred, if available. Tree top detection could be performed in a fully automatic manner with satisfying results, which was to be expected in an almost pure coniferous forest. Forest stand segmentation was also done fully automated and compared to a visual-manual delineation of forest stands. This comparison showed two main differences: first, the outlines of the automated segmentation are more detailed, which is necessary for an accurate estimation of the height parameters. Second, the automatic segmentation led to 
smaller and thus more segments than an interpreter would delineate. This fact is not a major drawback, since smaller segments can be merged in a final steps based on their calculated forest attributes and it is more important to generate homogeneous stands than to end up with too large and heterogeneous stands. Timber volume estimations are not yet finalized, preliminary results show lower correlation than expected and obtained in previous projects. Possible reasons include: (i) different behaviour in this alpine environment compared to lowland forests, (ii) changes in some to the forest stands between data acquisition and field work and (iii) an inconsistency in the field data, which has to be checked by an additional field campaign. This is also the outlook on what will be done next: additional field work to eliminate reasons (ii) and (iii) and then the roll-out of the process on large areas of Styria (approximately $2000 \mathrm{~km}^{2}$ ).

\subsection{References and/or Selected Bibliography}

References from Journals:

Alberti, G., Boscutti, F., Pirotti F., Bertacco C., De Simon G., Sigura M., Cazorzi F., Bonfanti, P., 2013. A LiDAR-based approach for a multi-purpose characterization of Alpine forests: an Italian case study. iForest (early view) - doi: 10.3832/ifor0876-006.

Hyyppä, J., Hyyppä, H., Yu, X., Kaartinen, H., Kukko, A., Holopainen, M., 2009. Forest Inventory Using Small Footprint Airborne Topographic Laser Ranging and Scanning Principles. In: Shan, J., Toth, C.K. (Eds.): CRC Press, Boca Raton, pp. 335-370.

Järnstedt, J. et al., 2012. Forest variables estimation using a high-resolution digital surface model. ISPRS Jour. of Photogr. and Rem.Sens., 74(2012), pp. 78-84.

Koch, B., 2010: Status and future of laser scanning, synthetic aperture radar and hyperspectral remote sensing data for forest biomass assessment, B. Koch / ISPRS Journal of Photogrammetry and Remote Sensing 65 (2010), pp. 581-590.

Lefsky, M.A., Cohen, W.B., Harding, D., Parker, G., Acker, S.A., Gower, S.T., 2001. Remote sensing of aboveground biomass in three biomes. International archives of the photogrammetry. Remote Sensing and Spatial Information Sciences 34 (Part 3/W4), pp. 155-160.

Nelson, R., Krabill, W., Tonelli, J., 1988. Estimating forest biomass and volume using airborne laser data. Remote Sensing of Environment 24 (2), pp. 247-267.

Popescu, S.C., 2007. Estimating biomass of individual pine trees using airborne LiDAR. Biomass and Bioenergy 31 (9), pp. 646-655.

Straub, C., Dees, M., Weinacker, H., Koch, B., 2009. Using airborne laser scanner data and CIR orthophotos to estimate the stem volume of forest stands. Photogrammetrie, Fernerkundung, Geoinformation 30 (3), pp. 277-287.

\section{References from Books:}

Gonzalez, R.C. and Woods, R.E. 2002. Digital Image Processing. Prentice Hall, Inc., Upper Saddle River, New Jersey, second edition, pp. 793.

\section{References from Other Literature:}

Gallaun H., Schardt M., Linser S. 2007. Remote Sensing based Forest Map of Austria and derived Environmental Indicators. ForestSat Conference, Montpellier, France, Nov. 5-7, 2007, 5 pages, published on CD.

Hirschmugl, M., 2008. Derivation of Forest Parameters from UltracamD Data. Dissertation. Technische Universität Graz, pp. 200.

Wack, R. and Stelzl, H., 2005. Assessment of forest stand parameters from laserscanner data in mixed forests. Proc. ForestSat 2005, Borås, pp. 56-60.

\subsection{Acknowledgements}

The authors acknowledge the co-funding of EUFODOS by the European Commission Seventh Framework Cooperation Programme, FP7-SPACE-2010-1. They wish to thank Dr. Gerhard Pelzmann from the Styrian Chamber of Agriculture for his input in ground truth data calculation and the "Land Steiermark" for their thematic support. 